\title{
Cognitive impairment in pre-eclampsia complicated by eclampsia and pulmonary oedema after delivery: a case control study
}

\author{
Lina Bergman ${ }^{1}$, Lilja Thorgeirsdottir ${ }^{2}$, Helen Elden ${ }^{1}$, Susanne Hesselman ${ }^{3}$, Sonja Schell ${ }^{4}$, \\ Evelina Ahlm ${ }^{5}$, Annet Aukes ${ }^{6}$, and Catherine Cluver ${ }^{4}$ \\ ${ }^{1}$ Sahlgrenska Academy \\ ${ }^{2}$ University of Gothenburg Sahlgrenska Academy \\ ${ }^{3}$ Uppsala Universitet \\ ${ }^{4}$ Stellenbosch University \\ ${ }^{5}$ Lund University \\ ${ }^{6}$ Radboud University Medical Center
}

October 6, 2020

\begin{abstract}
Objectives We aimed to assess cognitive function in women with pre-eclampsia stratified by severity, before and after onset of disease. Design Prospective case control study Setting Single center study at a referral hospital in Cape Town, South Africa. Population Pregnant women between 20 and 42 weeks gestation with eclampsia, pulmonary oedema, pre-eclampsia without severe features a normotensive pregnancy. Methods Women were included at diagnosis (cases) or at admission for delivery (controls). Two cognitive assessments, the Cognitive Failure Questionnaire (CFQ) to assess the cognitive function subjectively before inclusion in the study, and Montreal Cognitive Assessment (MoCA) to assess the current cognitive function objectively before discharge from the hospital after delivery were performed. Main outcome measures Total- and subscores from the CFQ and MoCa tests. Results We included 61 women with eclampsia, 28 with pre-eclampsia complicated by pulmonary oedema, 38 with pre-eclampsia without severe features and 26 with normotensive pregnancies. There was no difference in cognitive function from early pregnancy between groups. Women with eclampsia and pre-eclampsia complicated by pulmonary oedema scored lower on the MoCA assessment at time of discharge compared to women with normotensive pregnancies. The results were attenuated in pulmonary oedema after adjustment for confounders. Conclusion Women with pre-eclampsia complicated by pulmonary oedema and in particular eclampsia had impaired cognitive function after onset of disease compared to normotensive pregnant controls. The impairment did not seem to be present before onset of disease. Women with pre-eclampsia without severe features did not have impaired cognitive function.
\end{abstract}

Cognitive impairment in pre-eclampsia complicated by eclampsia and pulmonary oedema after delivery: a case control study

Lina BERGMAN, MD, PhD ${ }^{1,2}$, Lilja THORGEIRSDOTTIR, RNM, B.Sc ${ }^{3}$, Helen ELDEN, RNM, PhD ${ }^{2,3}$, Susanne HESSELMAN, MD, PhD ${ }^{4,5}$, Sonja SCHELL RNM ${ }^{1}$, Evelina AHLM, MS ${ }^{7}$, Annet AUKES, MD, $\mathrm{PhD}^{6}$ and Catherine CLUVER, $\mathrm{MD}, \mathrm{PhD}^{1,8}$

Cape Town, South Africa

${ }^{1}$ Department of Obstetrics and Gynecology, Stellenbosch University, Cape Town, South Africa

${ }^{2}$ Department of Obstetrics and Gynecology, Institute of clinical sciences, Sahlgrenska Academy, University of Gothenburg, Gothenburg, Sweden 
${ }^{3}$ Department of Reproductive and Perinatal health, Institute of Health and Care Sciences, Sahlgrenska Academy, University of Gothenburg, Gothenburg, Sweden

${ }^{4}$ Department of Women's and Children's Health, Uppsala University, Uppsala, Sweden

${ }^{5}$ Center for clinical research, Uppsala University, Falun, Sweden

${ }^{6}$ Department of Obstetrics and Gynecology, Amalia children's hospital, Radboud University Medical Center, Nijmegen, the Netherlands

${ }^{7}$ Department of Obstetrics and Gynecology, Faculty of Medicine, Lund University, Sweden

${ }^{8}$ Mercy Perinatal, Melbourne University, Melbourne, Australia

Conflicts of interest

The authors report no conflict of interest.

Corresponding author

Lina Bergman

Department of Obstetrics and Gynecology

PO Kvinnokliniken SU Östra SE 41685 Göteborg, Sweden

Telephone number 0046707920780, Email:lina.bergman.2@gu.se

Running title:

Cognitive impairment in severe pre-eclampsia

Word Count Abstract: 245

Word Count Main Text: 2974

ABSTRACT

\section{Objectives}

We aimed to assess cognitive function in women with pre-eclampsia stratified by severity, before and after onset of disease.

\section{Design}

Prospective case control study

\section{Setting}

Single center study at a referral hospital in Cape Town, South Africa.

\section{Population}

Pregnant women between 20 and 42 weeks gestation with eclampsia, pulmonary oedema, pre-eclampsia without severe features a normotensive pregnancy.

\section{Methods}

Women were included at diagnosis (cases) or at admission for delivery (controls). Two cognitive assessments, the Cognitive Failure Questionnaire (CFQ) to assess the cognitive function subjectively before inclusion in the study, and Montreal Cognitive Assessment (MoCA) to assess the current cognitive function objectively before discharge from the hospital after delivery were performed.

\section{Main outcome measures}

Total- and subscores from the CFQ and MoCa tests. 


\section{Results}

We included 61 women with eclampsia, 28 with pre-eclampsia complicated by pulmonary oedema, 38 with pre-eclampsia without severe features and 26 with normotensive pregnancies. There was no difference in cognitive function from early pregnancy between groups. Women with eclampsia and pre-eclampsia complicated by pulmonary oedema scored lower on the MoCA assessment at time of discharge compared to women with normotensive pregnancies. The results were attenuated in pulmonary oedema after adjustment for confounders.

\section{Conclusion}

Women with pre-eclampsia complicated by pulmonary oedema and in particular eclampsia had impaired cognitive function after onset of disease compared to normotensive pregnant controls. The impairment did not seem to be present before onset of disease. Women with pre-eclampsia without severe features did not have impaired cognitive function.

\section{Funding}

The study was supported by the Swedish Medical Society, Märta Lundqvist foundation and Mercy Perinatal. LB is supported by the Swedish Society for Medical Research (SSMF). SH is supported by Center for clinical research Dalarna, Uppsala University (grant CKFUU-744551) CC receives salary support from the Mercy Perinatal Foundation.

\section{Keywords}

Pre-eclampsia, eclampsia, pulmonary oedema, cognitive function

\section{TWEETABLE ABSTRACT}

Pre-eclampsia complicated by eclampsia or pulmonary oedema demonstrated an acute impairment on cognitive function.

\section{INTRODUCTION}

Pre-eclampsia, defined as hypertension with end-organ dysfunction after 20 weeks of gestation, is a multisystem disorder that complicates about $4-6 \%$ of all pregnancies. ${ }^{1,2}$ Cerebral and cardiovascular complications, which include eclampsia and pulmonary oedema, are some of the most severe outcomes and can result in maternal mortality and morbidity. ${ }^{3}$ Long-term neurological effects of pre-eclampsia and its complications include an increased risk for white matter lesions, stroke, seizure disorders and vascular dementia later in life. ${ }^{4-6}$ Women with previous pre-eclampsia have also reported a poorer quality of life and social functioning, and have an increased risk for post-traumatic stress disorder. ${ }^{7,8}$

The impact of pre-eclampsia and its complications on cognitive function is a field that requires further investigation. In 2018, a systematic review found that women with a history of pre-eclampsia reported subjective losses in cognitive function. They also noted that there was insufficient data to conclude about the presence or absence of objective cognitive changes. This review excluded women with a history of eclampsia and assessed all women together irrespective of time after delivery. ${ }^{9}$ Later in the same year, a large longitudinal cohort study found differences in raw neurocognitive scores when women with and without pre-eclampsia were compared decades after pregnancy. The differences were seen in the domains assessing psychomotor speed and executive function but not working memory, but after adjustment for possible confounders the associations were attenuated. ${ }^{10}$

Whether the impairment in cognitive function observed years after pre-eclampsia exists in closer proximity to the diagnosis of pre-eclampsia and its relation to severity of disease is uncertain. It is also not known whether women with pre-eclampsia have underlying cognitive function deficits before the diagnosis of pre-eclampsia. We therefore aimed to assess cognition subjectively and objectively with testing performed at discharge after delivery. 


\section{METHODS}

The study had ethical approval (protocol number N18/03/034, Federal Wide assurance number 00001372, Institutional Review Board number IRB0005239) and all included participants signed informed consent before being enrolled in the PROVE (Pre-eclampsia Obstetric Adverse Events) biobank. Three women previously affected by severe pre-eclampsia were consulted about the cognitive function tests and time of testing.

\section{Population}

We included women with pre-eclampsia and normotensive pregnancies who were recruited to the PROVE biobank and database at Tygerberg Hospital, Cape Town, South Africa. Tygerberg Hospital is a referral hospital that manages only high-risk pregnancies and delivers over 7000 women a year. The PROVE biobank is an ongoing collaborative project that recruits women with pre-eclampsia and control pregnancies to facilitate research in the field of pre-eclampsia and in particular pre-eclampsia with severe features, focusing on eclampsia and pulmonary oedema. The study is registered with ISCRTN, registration number ISRCTN10623443. PROVE collects the recommended predictors and core-outcome sets recommended by CoLab to facilitate data sharing in preeclampsia research. ${ }^{11}$

For this study, we included women with singleton pregnancies who were able to speak and understand English, Xhosa or Afrikaans. Exclusion criteria included known neurological or cardiac disease. For normotensive women, additional exclusion criteria were chronic hypertension and diabetes mellitus. We included women with eclampsia, pulmonary oedema (as an example of pre-eclampsia with severe features), pre-eclampsia without complications and normotensive pregnancies.

Pre-eclampsia was defined according to the NICE guidelines. ${ }^{12}$ Eclampsia was confirmed when pre-eclampsia was complicated by generalized tonic-clonic seizures in the absence of another etiology. Pulmonary oedema was diagnosed when there was worsening dyspnea, fine bibasal inspiratory crackles on auscultation and features of pulmonary oedema on chest x-ray. Women were considered to have pre-eclampsia without severe features if they did not meet the NICE guidelines classification of severe pre-eclampsia. ${ }^{12}$ Pregnancies were considered to be normotensive if the women consistently had a systolic blood pressure below $140 \mathrm{mmHg}$ and a diastolic blood pressure below $90 \mathrm{mmHg}$ during pregnancy. Hemolysis, Elevated Liver Enzymes and Low Platelets syndrome (HELLP) was defined according to NICE guidelines ${ }^{12}$ and renal impairment was defined as a serum creatinine above $120 \mathrm{umol} / \mathrm{L}$.

Baseline data were obtained by interview and extraction from medical records. All data were entered and stored using REDCap (Research Electronic Data Capture) tools hosted at Stellenbosch University. ${ }^{13,}{ }^{14}$ Data were double checked for accuracy and controlled with original data collection forms.

\section{Cognitive function testing}

Cognitive function was assessed using two instruments: the Cognitive Failure Questionnaire (CFQ) and the Montreal Cognitive Assessment (MoCA) test. The CFQ is a subjective questionnaire that assesses the likelihood of committing errors in the completion of daily tasks such as the routines of everyday life. ${ }^{15}$ The CFQ consists of 25 items that are scored on a 5-point scale (range, 0 [never] to 4 [very often]). Thus, the total score ranges from 0-100, with higher scores indicating more frequently occurring cognitive failures. Women included in this study were instructed to complete the items with specific reference to the past 6 months prior to admission to hospital to assess the cognitive function prior to onset of pre-eclampsia. In the CFQ, there are 4 subscales that pertain to more specific areas of cognitive failures. These subscales are memory $(7$ items; range, 0-28), distractibility (9 items; range, 0-36), blunders (7 items; range, 0-28) and names (2 items; range, $0-8) \cdot{ }^{15}$

The MoCA test is an objective assessment composed of a variety of cognitive domains assessing attention, concentration, executive function, memory, language, visio-spatial abilities, abstract thinking, mathematical calculations and orientation. ${ }^{16}$ The highest possible score is 30 points and a score of 26 points or greater indicates normal function in individuals with a total school education of more than 12 years. If total school education is less than 12 years, an additional point is added to the total score. ${ }^{16}$ 
The assessments were administered by trained research midwives and a trained medical student. All assessments were performed postpartum, as close to discharge as possible, to avoid situations where women could have been too tired or sick to perform adequately. The CFQ test was available in English and Afrikaans and questions were translated to Xhosa by an interpreter. The MoCA test was available in English and Afrikaans and performed in Xhosa if needed at time of assessment by the Xhosa speaking research midwife. Translations to Xhosa from English for both the CFQ test and the MoCa test were checked for accuracy during the first five tests in order to control that the women understood the questions correctly and to secure validity.

\section{Statistics}

Demographic and clinical characteristics were presented as means with standard deviations (SD) and percentages and were compared between groups by ANOVA and chi-square tests. MoCA and CFQ scores were presented as means with $95 \%$ confidence intervals (CI) and compared between groups by use of robust Oneway ANOVA (parameter estimates with robust standard error to adjust for differences in variance) and pairwise comparisons with Bonferroni correction. To control for potential confounders, a robust multi-Way ANOVA (parameter estimates with robust standard error to adjust for differences in variance) was conducted with maternal age, gestational age at birth, days from test to discharge and parity as continuous covariates and living conditions as a fixed effects and were presented as estimated marginal means with 95 $\%$ CI. Normotensive pregnancy was set as the reference group in pairwise comparisons. In all hypothesis tests, a p-value of less than 0.05 was considered statistically significant. Data and statistical analyses were performed using SPSS version 26.0 (SPSS; PASW statistics) for MAC software package.

Sample size

To our knowledge, CFQ has never been tested in close proximity to diagnosis of pre-eclampsia previously. Previous publications have demonstrated a mean score for women with eclampsia of 44 with an SD of 16 and for women with previously normal pregnancy a mean of 36 with an SD between 11-14 when testing has been conducted years after pregnancy. ${ }^{17,18}$ With an alpha value of 0.05 and power of 0.80 , the sample size would be 25-60 in each group. For the MoCa test there are, to the best of our knowledge, no publications in the field of pre-eclampsia.

\section{FUNDING}

The study was supported by the Swedish Medical Society, Märta Lundqvist foundation and Mercy Perinatal. LB is supported by the Swedish Society for Medical Research (SSMF). SH is supported by Center for clinical research Dalarna, Uppsala University (grant CKFUU-744551) CC receives salary support from the Mercy Perinatal Foundation.

\section{RESULTS}

\section{Participants}

This study was conducted from April 2018 until November 2019 during which 222 women were included into the PROVE biobank. Fifty-one women did not have cognitive function assessments as they were included in the biobank for other sub-studies. One hundred seventy-one women had assessments for cognitive function. Eleven were excluded due to language barriers. Seven women from the group of pre-eclampsia without severe features were excluded as they developed renal impairment and/or HELLP. Of the 153 included in the study, 61 had eclampsia, 28 had pre-eclampsia complicated by pulmonary oedema, 38 had pre-eclampsia without severe complications and 26 had normotensive pregnancies (Figure 1). The majority had both subjective $(\mathrm{n}=140)$ and objective $(\mathrm{n}=139)$ assessments performed. Both CFQ and MoCA tests were administered as close to discharge as possible and none of the participants were treated with magnesium sulphate at time of testing.

\section{Background characteristics}


Maternal characteristics and pregnancy outcomes are presented in Table 1. Women with eclampsia were generally younger, less often cohabitating, more often registered as students, more commonly nulliparous and more likely to smoke. Women with pulmonary oedema were more commonly of black race, living in informal settlements, had shorter gestations and higher rates of termination of pregnancy before viability due to severe disease. Sixteen $(26 \%)$ women with eclampsia and $6(18 \%)$ women with pulmonary oedema suffered from HELLP. Eleven (18\%) of women with eclampsia and 4 (14\%) of women with pulmonary oedema developed renal impairment. None of the women had both eclampsia and pulmonary oedema.

\section{CFQ assessment}

There were no differences in the CFQ assessments between the groups before or after adjustment for potential confounders (Table 2). All groups scored similar in the four subdomains of memory, distractibility, blunders and names. (Table 3).

\section{MoCA assessment}

MoCA scores were lower in women with eclampsia and pulmonary oedema (21.6, 95\% CI 20.4 to 22.7 , $\mathrm{p}<0.001$ and $21.3,95 \%$ CI 19.7 to $23.0, \mathrm{p}<0.01$ respectively) when compared to normotensive women (25.8, 95\% CI 24.1 to 27.4). When adjusted for confounders, results remained similar for women with eclampsia $(21.7,95 \%$ CI 20.5 to 22.9 vs $25.6,95 \%$ CI 23.7 to $27.4, \mathrm{p}<0.05)$ (Table 2). For women with pulmonary oedema, the results were attenuated after adjustments for confounders (21.8, 95\% CI 20.0 to 23.5 vs 25.6, $95 \%$ CI 23.7 to $27.4, \mathrm{p}=0.09$ ). In women with eclampsia and women with pulmonary oedema, more than $75 \%$ had a score less than 26 (40/61 (76\%) for pulmonary oedema and 21/28 (81\%) for eclampsia). In women with pre-eclampsia without severe features, $8 / 45(18 \%)$ had a score less than 26 and in the normotensive group, $3 / 26(12 \%)$ had a score less than 26. For the subdomains of the MoCA assessment, there was an overall difference in attention, language, abstraction, delayed recall and orientation but no difference in naming or visuospatial ability (Table 4).

\section{DISCUSSION}

\section{Main findings}

When women were asked to assess their cognitive function subjectively over the six months prior to delivery (corresponding to early pregnancy), there were no differences in the overall or subdomain scores for any of the groups. At discharge, after delivery, women with pre-eclampsia complicated by pulmonary oedema or eclampsia demonstrated lower scores on the objective MoCA assessment for cognitive function when compared to normotensive women but the difference was attenuated for women with pulmonary oedema after adjustment for confounders. Women with pre-eclampsia without severe features showed no difference on MoCA scores when compared to normotensive women.

\section{Interpretation}

Previous studies have shown cognitive decline months to years after a pregnancy complicated by preeclampsia. ${ }^{17-19}$ To our knowledge no studies have assessed cognitive function before delivery or assessed cognitive function objectively at time of disease. Our findings would imply that the cognitive decline observed postpartum in earlier studies does not exist before onset of disease and is reported only in pre-eclampsia with severe features after diagnosis. To support this theory, subjective cognitive decline seems to increase by severity of disease and in particular by number of fits in women with previous eclampsia, arguing for a dose-response mechanism. ${ }^{17}$ It would be important to follow up our findings with long term studies to assess if these acute findings found in our population are reversible and if they correlate with longer term impaired cognitive function months to years after the pregnancy. In addition, cognitive function needs to be studied in combination with cerebral imaging and more in-depth cognitive function assessment on shortand long-term.

The CFQ was developed to assess cognitive function using daily life activities and does not have a cut off for normal function. It is recommended to perform the $\mathrm{CFQ}$ in generally comparable groups and to compare 
scores on a group level. The CFQ is a retrospective instrument subject to the limitations of human memory. It has been reported to change after severe physical stress or trauma like a brain injury and might be affected by a severe disease such as pre-eclampsia and in addition, CFQ has been designed to be used in a high income setting. ${ }^{20}$ Yet, the CFQ is the most commonly used assessment for cognitive function after pre-eclampsia. ${ }^{19,} 21,22$ In our study, many of the participants live in poverty and some of the items on the CFQ were not applicable. Examples include questions such as 'Do you find yourself forgetting why you went from one part of/room in the house to the other'. Many of the women in our study live in a single room where they sleep and eat. 'Do you find yourself forgetting what you came to the shop to buy?' was also problematic as many answered that they always only buy white bread. Although this may have influenced the outcomes, the groups were comparable.

The MoCA test was also developed in a high income setting and a score of 26 points is the cut off for normal cognitive function. ${ }^{16}$ In our population, even though women with normotensive pregnancies and non-complicated pre-eclampsia scored higher, the mean scores in these groups were 25.8 and 26.1 points respectively which correlates to borderline normal cognitive function. In a cross sectional study examining 370 healthy 18 year old South African males and females, the optimal cut off for sensitivity and specificity to detect cognitive impairment through the MoCA test was 24 points. ${ }^{23}$ Thus, women with normotensive pregnancies and non-complicated pre-eclampsia in our study scored above the suggested cut off for cognitive impairment whereas women with severe pre-eclampsia and in particular eclampsia scored below the limit of normal cognitive function. Many of the women with pre-eclampsia with severe features, including all those with eclampsia, were treated with magnesium sulphate for neuroprotection during their hospital stay. Magnesium sulphate has been shown to improve cognitive function in pregnant women. ${ }^{24}$ However, magnesium sulphate was not administered at the time of testing and scores were generally lower in women that had undergone treatment with magnesium sulphate (predominately women with eclampsia).

\section{Strengths and limitations}

This study included large numbers of women with severe disease which enabled us to assess different phenotypes of pre-eclampsia. To perform assessments in women with severe disease such as eclampsia is difficult in high income countries as the incidence is low, around 50/110,000. ${ }^{25}$ Performing this study in our setting, where eclampsia is more prevalent, enabled us to recruit this cohort in a short time period. Validated tests which included both subjective and objective cognitive function assessments were used. We also assessed cognition before and after delivery using different methods. To our knowledge, this study is the first to examine cognitive function in close proximity to onset of pre-eclampsia.

Our study does have limitations. Women with pre-eclampsia with severe features may have been more tired at the time of cognitive function testing. We tried to correct for this by adjusting for "time from delivery to discharge" in the analyses. In addition, no testing for anxiety or depression was performed. Though, very few women reported symptoms suggestive of depression during pregnancy and we adjusted for gestational age as a proxy for maternal and neonatal complications that could be related to anxiety and depression postpartum. The group of women with pulmonary oedema was small, introducing the risk of a type 2 error in the analyses where the differences in MoCA test were attenuated after adjustment for confounders. Lastly, there was no follow up after discharge. Women who deliver at Tygerberg hospital often live in informal settlements and it is challenging for them to return for follow up visits due to social circumstances. Finally, women rated their cognitive function prior to diagnosis or delivery retrospectively when doing the CFQ assessment that could introduce bias. However, the CFQ is the most common test used in the field and our results compare to previously published studies.

\section{Conclusion}

Pre-eclampsia with severe features such as eclampsia and pulmonary oedema may inflict an insult on the brain resulting in poorer cognitive performance in close proximity to the onset of disease. These findings need to be confirmed in other populations and in greater detail. Further research is also needed to assess whether these findings are reversible or if they persist and worsen. The cognitive impairment now found at the time 
of a severe hypertensive disorder in pregnancy might play a role in the pathway towards the development of cerebrovascular diseases such as stroke and vascular dementia in these women.

\section{ACKNOWLEDGMENTS}

We thank Zukiswa Magogotye for performing cognitive function assessments.

\section{DISCLOSURE OF INTERESTS}

The authors declare no conflict of interest.

\section{CONTRIBUTION TO AUTHORSHIP}

LB and CC planned, conducted and funded the study. SS and EA recruited the women in the study and performed the cognitive function tests. LB, CC, AA, HE, LT and SH analyzed the data. LB, LT and SH performed the statistical analyses. LB wrote the initial draft that was edited by CC and AA. All co-authors read, commented and approved the final version of the manuscript.

\section{DETAILS OF ETHICS APPROVAL}

The study had ethical approval (protocol number N18/03/034, Federal Wide assurance number 00001372, Institutional Review Board number IRB0005239) and all included participants signed informed consent before being enrolled in the PROVE (Pre-eclampsia Obstetric Adverse Events) biobank. The study was initially approved on the $28^{\text {th }}$ of February 2018 with annual progress reports approved yearly.

\section{FUNDING}

The study was supported by the Swedish Medical Society, Märta Lundqvist foundation and Mercy Perinatal. LB is supported by the Swedish Society for Medical Research (SSMF). SH is supported by Center for clinical research Dalarna, Uppsala University (grant CKFUU-744551) CC receives salary support from the Mercy Perinatal Foundation.

\section{REFERENCES}

1. Mol BWJ, Roberts CT, Thangaratinam S, Magee LA, de Groot CJM, Hofmeyr GJ. Pre-eclampsia. Lancet. 2016 Mar 05;387(10022):999-1011.

2. Abalos E, Cuesta C, Grosso AL, Chou D, Say L. Global and regional estimates of preeclampsia and eclampsia: a systematic review. European journal of obstetrics, gynecology, and reproductive biology. 2013 Sep;170(1):1-7.

3. Duley L. The global impact of pre-eclampsia and eclampsia. Semin Perinatol. 2009 Jun;33(3):130-7.

4. Basit S, Wohlfahrt J, Boyd HA. Pre-eclampsia and risk of dementia later in life: nationwide cohort study. BMJ. 2018 Oct 17;363:k4109.

5. Nerenberg KA, Park AL, Vigod SN, Saposnik G, Berger H, Hladunewich MA, et al. Long-term Risk of a Seizure Disorder After Eclampsia. Obstet Gynecol. 2017 Dec;130(6):1327-33.

6. McDonald SD, Malinowski A, Zhou Q, Yusuf S, Devereaux PJ. Cardiovascular sequelae of preeclampsia/eclampsia: a systematic review and meta-analyses. American heart journal. 2008 Nov;156(5):918-30.

7. Postma IR, Slager S, Kremer HP, de Groot JC, Zeeman GG. Long-term consequences of the posterior reversible encephalopathy syndrome in eclampsia and preeclampsia: a review of the obstetric and nonobstetric literature. Obstetrical \& gynecological survey. 2014 May;69(5):287-300.

8. Porcel J, Feigal C, Poye L, Postma IR, Zeeman GG, Olowoyeye A, et al. Hypertensive disorders of pregnancy and risk of screening positive for Posttraumatic Stress Disorder: A cross-sectional study. Pregnancy Hypertens. 2013 Oct;3(4):254-60. 
9. Elharram M, Dayan N, Kaur A, Landry T, Pilote L. Long-Term Cognitive Impairment After Preeclampsia: A Systematic Review and Meta-analysis. Obstet Gynecol. 2018 Aug;132(2):355-64.

10. Dayan N, Kaur A, Elharram M, Rossi AM, Pilote L. Impact of Preeclampsia on Long-Term Cognitive Function. Hypertension. 2018 Dec;72(6):1374-80.

11. Myatt L, Redman CW, Staff AC, Hansson S, Wilson ML, Laivuori H, et al. Strategy for standardization of preeclampsia research study design. Hypertension. 2014 Jun;63(6):1293-301.

12. NICE. Hypertension in pregnancy: diagnosis and management. 2019 [cited 2020-09-22]; Available from:www.nice.org.uk/guidance/ng133

13. Harris PA, Taylor R, Thielke R, Payne J, Gonzalez N, Conde JG. Research electronic data capture (REDCap)-a metadata-driven methodology and workflow process for providing translational research informatics support. J Biomed Inform. 2009 Apr;42(2):377-81.

14. Harris PA, Taylor R, Minor BL, Elliott V, Fernandez M, O'Neal L, et al. The REDCap consortium: Building an international community of software platform partners. J Biomed Inform. 2019 Jul;95:103208.

15. Broadbent DE, Cooper PF, FitzGerald P, Parkes KR. The Cognitive Failures Questionnaire (CFQ) and its correlates. Br J Clin Psychol. 1982 Feb;21 (Pt 1):1-16.

16. Nasreddine ZS, Phillips NA, Bedirian V, Charbonneau S, Whitehead V, Collin I, et al. The Montreal Cognitive Assessment, MoCA: a brief screening tool for mild cognitive impairment. J Am Geriatr Soc. 2005 Apr;53(4):695-9.

17. Aukes AM, Wessel I, Dubois AM, Aarnoudse JG, Zeeman GG. Self-reported cognitive functioning in formerly eclamptic women. Am J Obstet Gynecol. 2007 Oct;197(4):365 e1-6.

18. Postma IR, Bouma A, de Groot JC, Aukes AM, Aarnoudse JG, Zeeman GG. Cerebral white matter lesions, subjective cognitive failures, and objective neurocognitive functioning: A follow-up study in women after hypertensive disorders of pregnancy. J Clin Exp Neuropsychol. 2016;38(5):585-98.

19. Baecke M, Spaanderman ME, van der Werf SP. Cognitive function after pre-eclampsia: an explorative study. J Psychosom Obstet Gynaecol. 2009 Mar;30(1):58-64.

20. Dockree PM, O'Keeffe FM, Moloney P, Bishara AJ, Carton S, Jacoby LL, et al. Capture by misleading information and its false acceptance in patients with traumatic brain injury. Brain. 2006 Jan;129(Pt 1):12840.

21. Aukes AM, Vitullo L, Zeeman GG, Cipolla MJ. Pregnancy prevents hypertensive remodeling and decreases myogenic reactivity in posterior cerebral arteries from Dahl salt-sensitive rats: a role in eclampsia? American journal of physiology Heart and circulatory physiology. 2007 Feb;292(2):H1071-6.

22. Postma IR, Groen H, Easterling TR, Tsigas EZ, Wilson ML, Porcel J, et al. The brain study: Cognition, quality of life and social functioning following preeclampsia; An observational study. Pregnancy Hypertens. 2013 Oct;3(4):227-34.

23. Beath N, Asmal L, van den Heuvel L, Seedat S. Validation of the Montreal cognitive assessment against the RBANS in a healthy South African cohort. S Afr J Psychiatr. 2018;24:1304.

24. Rana S, Lindheimer M, Hibbard J, Pliskin N. Neuropsychological performance in normal pregnancy and preeclampsia. Am J Obstet Gynecol. 2006 Jul;195(1):186-91.

25. Kullberg G, Lindeberg S, Hanson U. Eclampsia in Sweden. Hypertension in pregnancy : official journal of the International Society for the Study of Hypertension in Pregnancy. 2002;21(1):13-21.

Figure legends

Figure 1 . Flow chart describing the study population. 
Table 1. Maternal characteristics and pregnancy outcomes of the study population.

\begin{tabular}{|c|c|c|c|}
\hline & Eclampsia & Pulmonary oedema & Pre-eclampsia without severe features \\
\hline $\mathbf{n}$ & 61 & 28 & 38 \\
\hline Maternal age, years (SD) & $22.5(6)$ & $27.6(7)$ & $25.7(6)$ \\
\hline Race, $\mathbf{n}(\%)$ & 61 & 28 & 38 \\
\hline Black & $40(66)$ & $21(75)$ & $26(62)$ \\
\hline Colored & $21(34)$ & $7(25)$ & $12(38)$ \\
\hline Caucasian & $0(0)$ & $0(0)$ & $0(0)$ \\
\hline Marital status, n (\%) & 61 & 28 & 38 \\
\hline Cohabitating & $31(51)$ & $20(71)$ & $19(50)$ \\
\hline Single & $30(49)$ & $8(29)$ & $19(50)$ \\
\hline Education & 61 & 28 & 38 \\
\hline years (SD) & $10.6(2)$ & $10.8(2)$ & $11.1(2)$ \\
\hline$>10$ years education $\mathrm{n}(\%)$ & $36(59)$ & $17(61)$ & $29(76)$ \\
\hline$>16$ years, years $(\mathrm{SD})$ & $10.9(1)$ & $10.9(2)$ & $11.1(2)$ \\
\hline Job situation, n (\%) & 61 & 28 & 38 \\
\hline Working & $16(26)$ & $12(43)$ & $14(37)$ \\
\hline Student & $18(30)$ & $2(7)$ & $8(21)$ \\
\hline Unemployed & $27(44)$ & $14(50)$ & $16(42)$ \\
\hline Living conditions, $\mathrm{n}(\%)$ & 61 & 28 & 38 \\
\hline House or apartment & $34(56)$ & $14(50)$ & $23(61)$ \\
\hline Informal settlement & $27(44)$ & $14(50)$ & $15(39)$ \\
\hline Nulliparous, n (\%) & $43(71)$ & $10(36)$ & $20(53)$ \\
\hline HIV positive, n (\%) & $7(12)$ & $6(21)$ & $6(16)$ \\
\hline Smoker, n (\%) & $11(18)$ & $2(7)$ & $1(3)$ \\
\hline Alcohol use, n (\%) & $7(12)$ & $0(0)$ & $1(3)$ \\
\hline Metamphetamine use, n (\%) & $3(6)$ & $1(5)$ & $0(0)$ \\
\hline Diabetes, n (\%) & $0(0)$ & $0(0)$ & $1(2.7)$ \\
\hline Chronic hypertension, n (\%) & $4(7)$ & $2(7)$ & $6(16)$ \\
\hline Anemia, n (\%) & $0(0)$ & $1(5)$ & $3(7.9)$ \\
\hline Depression, n (\%) & $2(4)$ & $0(0)$ & $2(5.3)$ \\
\hline GA at delivery, weeks (SD) & $33.1(5)$ & $31.6(5)$ & $34.0(4)$ \\
\hline Mode of delivery, n (\%) & 61 & 28 & 38 \\
\hline Vaginal delivery & $23(38)$ & $7(25)$ & $13(34)$ \\
\hline Planned caesarian section & $0(0)$ & $2(7)$ & $2(5)$ \\
\hline Emergency caesarian section & $38(62)$ & $19(68)$ & $23(61)$ \\
\hline Postpartum hemorrhage, n (\%) & $9(15)$ & $2(7)$ & $3(8)$ \\
\hline Coagulation disorder (\%) & $8(13)$ & $2(7)$ & $1(3)$ \\
\hline Delivery-discharge, days (SD) & $8.0(5)$ & $9.0(5)$ & $4.2(3)$ \\
\hline Other organ impairment, $\mathrm{n}(\%)$ & 61 & 28 & 38 \\
\hline HELLP & $16(26)$ & $5(18)$ & $0(0)$ \\
\hline Renal impairment & $11(18)$ & $4(14)$ & $0(0)$ \\
\hline Dialysis & $1(2)$ & $1(4)$ & $0(0)$ \\
\hline Neonatal outcome (\%) & 61 & 28 & 38 \\
\hline Discharged home & $20(33)$ & $8(29)$ & $17(45)$ \\
\hline Transferred to neonatal unit & $28(46)$ & $13(46)$ & $17(45)$ \\
\hline Termination of pregnancy & $5(8)$ & $5(18)$ & $3(8)$ \\
\hline Stillborn & $8(13)$ & $2(7)$ & $1(3)$ \\
\hline
\end{tabular}


Values are presented as means (standard deviation) and numbers (percentage).

GA; gestational age, HIV; Human Immunodeficiency Virus, SD; standard deviation

Table 2. Results of cognitive assessments (CFQ and MoCA) of women with eclampsia, pulmonary oedema and pre-eclampsia without severe features compared to women with normotensive pregnancies.

\begin{tabular}{|c|c|c|c|c|c|c|c|c|}
\hline & & CFQ $(95 \%$ CI $)$ & $P$-value & CFQ $(95 \%$ CI $)$ & $P$-value & & MoCA $(95 \%$ CI $)$ & $P$-value \\
\hline & & Mean score & & EMM & & $\mathrm{n}=$ & Mean score & \\
\hline & & & 0.45 & & 0.54 & & & $<0.001$ \\
\hline Eclampsia & 56 & $33.7(29.9,37,5)$ & $>0.99^{*}$ & $32.3(28.1,36.5)$ & $>0.99^{*}$ & 53 & $21.6(20.4,22.7)$ & $<0.001^{*}$ \\
\hline Pulmonary oedema & 25 & $28.5(22.8,34.2)$ & $>0.99^{*}$ & $27.3(21.2,33.4)$ & $>0.99 *$ & 26 & $21.3(19.7,23.0)$ & $<0.01^{*}$ \\
\hline Pre-eclampsia** & 34 & $31.5(26.6,36.4)$ & $>0.99^{*}$ & $32.7(28.1,37.8)$ & $>0.99^{*}$ & 35 & $26.4(25.0,27.8)$ & $>0.99^{*}$ \\
\hline Normotensive & 25 & $30.1(24.4,35.8)$ & - & $31.5(24.6,38.4)$ & - & 25 & $25.8(24.1,27.4)$ & - \\
\hline
\end{tabular}

Values are presented as means and estimated marginal means with $95 \%$ confidence intervals (CI). Results retrieved from robust one-way ANOVA and robust multi-way ANOVA with maternal age, gestational age at delivery, days from delivery to discharge and parity as continuous covariates and living condition as fixed effect for EMM.

*Pairwise comparisons versus normotensive with Bonferroni correction.

**Pre-eclampsia without severe features

CFQ; Cognitive Failure Questionnaire, EMM; Estimated Marginal Means, MoCA; Montreal Cognitive Assessment

Table 3. Results of subdomains of the CFQ in women with eclampsia, pulmonary oedema and pre-eclampsia without severe features compared to women with normotensive pregnancies.

\begin{tabular}{llllll}
\hline & $\mathrm{n}=$ & Memory & Distractibility & Blunders & Names \\
\hline P-value & & 0.366 & 0.303 & 0.764 & 0.818 \\
Eclampsia & 56 & $9.2(7.7,10.7)$ & $13.5(12.0,15.1)$ & $9.4(8.2,10.7)$ & $2.5(2.0,3.1)$ \\
Pulmonary oedema & 25 & $7.2(5.3,9.1)$ & $11.1(10.9,14.8)$ & $8.3(6.5,10.1)$ & $2.6(1.8,3.7)$ \\
Pre-eclampsia* & 34 & $8.4(7.0,9.8)$ & $12.9(8.0,17.0)$ & $9.4(7.8,10.9)$ & $2.2(1.5,2.9)$ \\
Normotensive & 25 & $7.9(5.7,10.1)$ & $11.8(9.5,14.1)$ & $9.0(7.2,10.8)$ & $2.2(1.4,3.0)$ \\
\hline
\end{tabular}

Values are presented as means with $95 \%$ confidence intervals. Results retrieved from robust ANOVA.

CFQ; Cognitive Failure Questionnaire, IQR; interquartile range,

*Pre-eclampsia without severe features

Table 4. Results of subdomains of MoCA in women with eclampsia, pulmonary oedema and pre-eclampsia without severe features compared with women with normotensive pregnancies.

\begin{tabular}{lllllllll}
\hline & $\mathrm{n}=$ & Visuospatial & Naming & Attention & Language & Abstraction & Delayed recall & $\mathrm{O}$ \\
\hline P-value & & 0.059 & 0.151 & $<0.001$ & $<0.001$ & $<0.01$ & $<0.001$ & $<$ \\
Eclampsia & 53 & $3.3(3.0,3.6)$ & $2.4(2.2,2.6)$ & $4.1(3.8,4.5)$ & $1.4(1.1,1.6)$ & $1.1(0.9,1.3)$ & $3.0(2.6,3.5)$ & 5. \\
Pulmonary oedema & 26 & $3.5(3.1,4.0)$ & $2.2(1.9,2.5)$ & $3.9(3.4,4.3)$ & $1.5(1.2,1.9)$ & $1.2(0.9,1.5)$ & $2.7(2.0,3.3)$ & 5. \\
Pre-eclampsia* & 35 & $4.0(3.6,4.3)$ & $2.7(2.4,2.9)$ & $5.2(4.8,5.7)$ & $2.3(2.0,2.7)$ & $1.6(1.3,1.9)$ & $4.2(3.7,4.8)$ & 5. \\
Normotensive & 25 & $3.8(3.4,4.2)$ & $2.5(2.2,2.8)$ & $5.1(4.6,5.6)$ & $2.4(2.1,2.8)$ & $1.5(1.2,1.8)$ & $3.8(3.1,4.4)$ & 5. \\
\hline
\end{tabular}


Values are presented as means with $95 \%$ confidence intervals. Results retrieved from robust ANOVA MoCA; Montreal Cognitive Assessment

*Pre-eclampsia without severe features

\section{Hosted file}

Figure 1 .pptx available at https://authorea.com/users/364804/articles/485097-cognitiveimpairment-in-pre-eclampsia-complicated-by-eclampsia-and-pulmonary-oedema-afterdelivery-a-case-control-study 